\title{
Isolated proximal greater saphenous vein thrombosis and the risk of propagation to deep vein thrombosis and pulmonary embolism
}

This article was published in the following Dove Press journal: Vascular Health and Risk Management

\author{
Samuel H Kim' \\ Nimesh Patel ${ }^{2}$ \\ Kanika Thapar ${ }^{2}$ \\ Ananda $V$ Pandurangadu ${ }^{3}$ \\ Amit Bahl' \\ 'Department of Emergency Medicine, \\ William Beaumont Hospital, Royal \\ Oak, MI, USA; ${ }^{2}$ Oakland University \\ William Beaumont School of \\ Medicine, Rochester, MI, USA; \\ ${ }^{3}$ Department of Emergency Medicine, \\ Rush University, Chicago, IL, USA
}

Objectives: Greater saphenous vein (GSV) thrombosis is concerning due to its close proximity to the deep femoral vein. This study sought to identify the risk of propagation to deep vein thrombosis (DVT) or pulmonary embolism (PE) among patients with isolated proximal GSV superficial thrombosis and describe provider practice patterns related to treatment.

Materials and methods: This is an Institutional Review Board-approved retrospective multicenter study. Patients presented to one of three possible emergency departments in a large health system. About 21,716 patients were queried through the electronic medical record. Ninety-five patients or $0.4 \%$ of study subjects met inclusion criteria of isolated proximal GSV thrombosis. Forty-five patients were excluded, leaving a final data set of 40 patients. Investigators recorded radiology impressions, patient demographics, past medical history, DVT/PE risk factors, and treatment plans. Propagation of GSV thrombosis to DVT/PE was also noted. Follow-up methods included chart review, primary care physician follow-up, and direct, scripted patient follow-up phone calls. Descriptive statistics were applied to study subjects using SAS for Windows, version 9.3.

Results: Three patients (7.5\%) had progression of GSV thrombosis to DVT/PE. Twenty percent of patients without malignancy were treated with anticoagulation compared to $14 \%$ of those with preexisting malignancy upon initial diagnosis of isolated GSV thrombosis. Forty-five percent of patients were prescribed some type of supportive therapy to aid in the treatment of GSV thrombosis.

Conclusion: Isolated proximal GSV thrombosis, while uncommon, may frequently progress to DVT or PE. Our work suggests clinicians should consider anticoagulation for isolated GSV thrombosis.

Keywords: greater saphenous vein thrombosis, superficial vein thrombosis, venous thromboembolism

\section{Introduction}

Early identification and treatment of deep vein thrombosis (DVT) remains an important medical issue due to complications of post-thrombotic syndrome (eg, pain, swelling) and pulmonary embolism (PE). Evidenced-based guidelines have been well established for this disease continuum. ${ }^{1-6}$ Superficial venous thrombophlebitis (SVT), on the other hand, is a common, yet overlooked and seemingly benign medical condition that can have serious complications. SVT of the lower limbs is specifically thought to be a self-limiting benign medical. ${ }^{5,7-9}$ As such, physicians have routinely treated SVT using widely variable approaches including but not limited to compression stockings, encouraging ambulation, aspirin, and nonsteroidal anti-inflammatory drugs. ${ }^{7,10}$ In contrast, DVTs are often treated aggressively using anticoagulants for at least 3 months,
Correspondence: Amit Bahl

Department of Emergency Medicine 360 I W I 3 Mile Road, Royal Oak, MI 48073, USA

Tel +l 2482290736

Email Amit.bahl@beaumont.edu 
due to significant risk for cardiac, pulmonary, and neurologic complications via their embolization. ${ }^{3,6,11}$

SVTs have an estimated incidence rate in the general population between 3\%-11\%, with the greater saphenous vein (GSV) disproportionately affected $60 \%-80 \%$ of the time. ${ }^{12,13}$ Thrombosis of the GSV presents clinically with warmth, edema, and palpable subcutaneous cords in the affected limb. ${ }^{39}$ Similar to DVTs/PEs, risk factors for SVT include advanced age, smoking, obesity, neoplasms, thromboembolic episodes, pregnancy, oral contraceptives, hormone replacement therapy, recent surgery, and autoimmune diseases. ${ }^{5,13,14}$ Furthermore, SVTs have a two-to-three time greater prevalence rate as compared to DVTs and PEs combined. ${ }^{15}$

SVTs in the proximal GSV are an area of concern and the focus of our research. The proximity of the GSV to the saphenofemoral junction (SFJ) makes SVTs a serious concern as there is the possibility of these clots dislodging and entering the deep venous system. ${ }^{16,17}$ Figure 1 depicts sonographic images of the SFJ patient and with clot. Our aging population with their multiple comorbidities makes them at an increased risk for SVT complications. ${ }^{10,18}$ The SFJ has recently been the focus of multiple studies that have suggested its possible role in facilitating extension of SVT into the common femoral vein, thereby causing DVT/PE. ${ }^{19-21}$ Thrombi below the knee in the GSV generally are considered clinically insignificant and do not typically warrant aggressive treatment. ${ }^{22}$ However, for thrombi that extend proximally to the SFJ, studies suggest up to $33 \%$ of these patients can develop PEs. ${ }^{12,23}$ The significance of thrombi in the SFJ and the risk of subsequent PE or DVT and the surrounding practice patterns remains inconsistent. ${ }^{22}$
Previous research noted that patients with SVTs often had concomitant DVTs, and asymptomatic and symptomatic PEs up to a rate of $44 \%, 33 \%$, and $13 \%$, respectively. ${ }^{12,19,20,23}$ However, these studies failed to establish this observation as independent events or a pattern of disease progression from SVT to DVT/PE at the time of imaging.

The focus of this study was to:

1. Identify risk of propagation/conversion to DVT/PE among isolated proximal GSV SVTs

2. Describe provider practice patterns related to treatment of proximal GSV SVTs

3. Describe current usage of anticoagulation for proximal GSV SVTs

\section{Materials and methods}

This was a retrospective multicenter study of three sites with approval from the Beaumont Health Institutional Review Board. Patients presented to one of three possible emergency departments in a large health system which included a Level I Trauma tertiary care facility, a Level II Trauma community emergency department, and a mid-size community emergency department. Patients were initially queried through the electronic medical record EPIC over a 2-year period from December 11, 2013, to December 31, 2015 , for duplex Doppler ultrasound of the lower extremity veins performed on unilateral and bilateral superficial and deep venous structures, with 21,716 patients identified. No written informed consent was obtained prior to the review of the medical records. Figure 2 demonstrates the pathway of inclusions/exclusions for this study. After

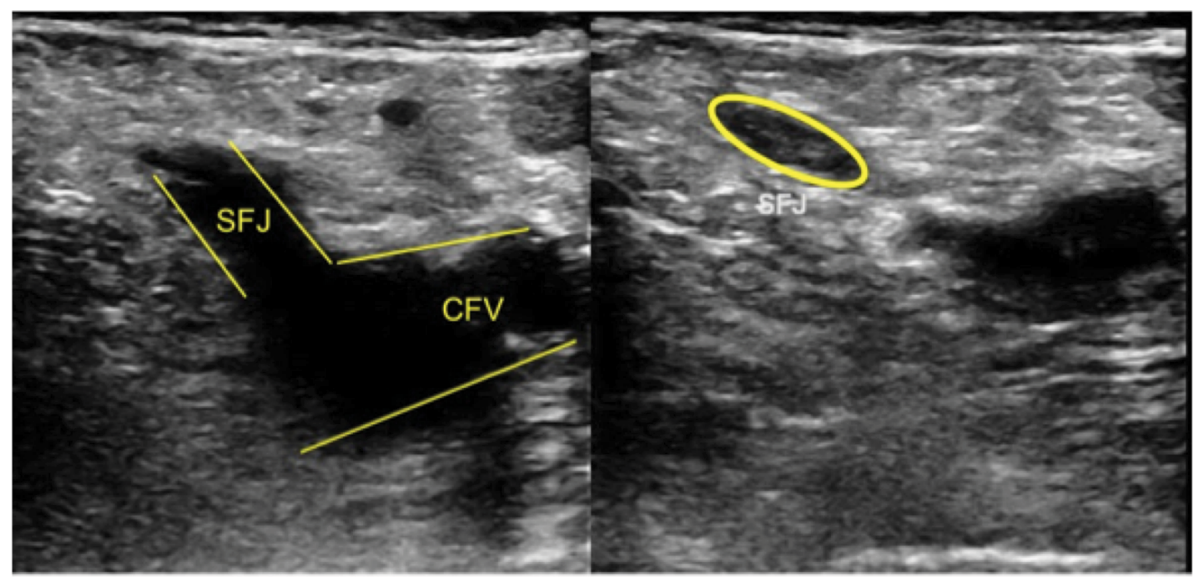

Figure I Demonstration of superficial venous thrombus at the proximal GSV by venous duplex compression ultrasonography.

Notes: The figure shows a noncompressible GSV which indicates the presence of a venous thrombus within the greater saphenous vein near the SFJ. Abbreviations: CFV, common femoral vein; GSV, greater saphenous vein; SFJ, saphenofemoral junction. 


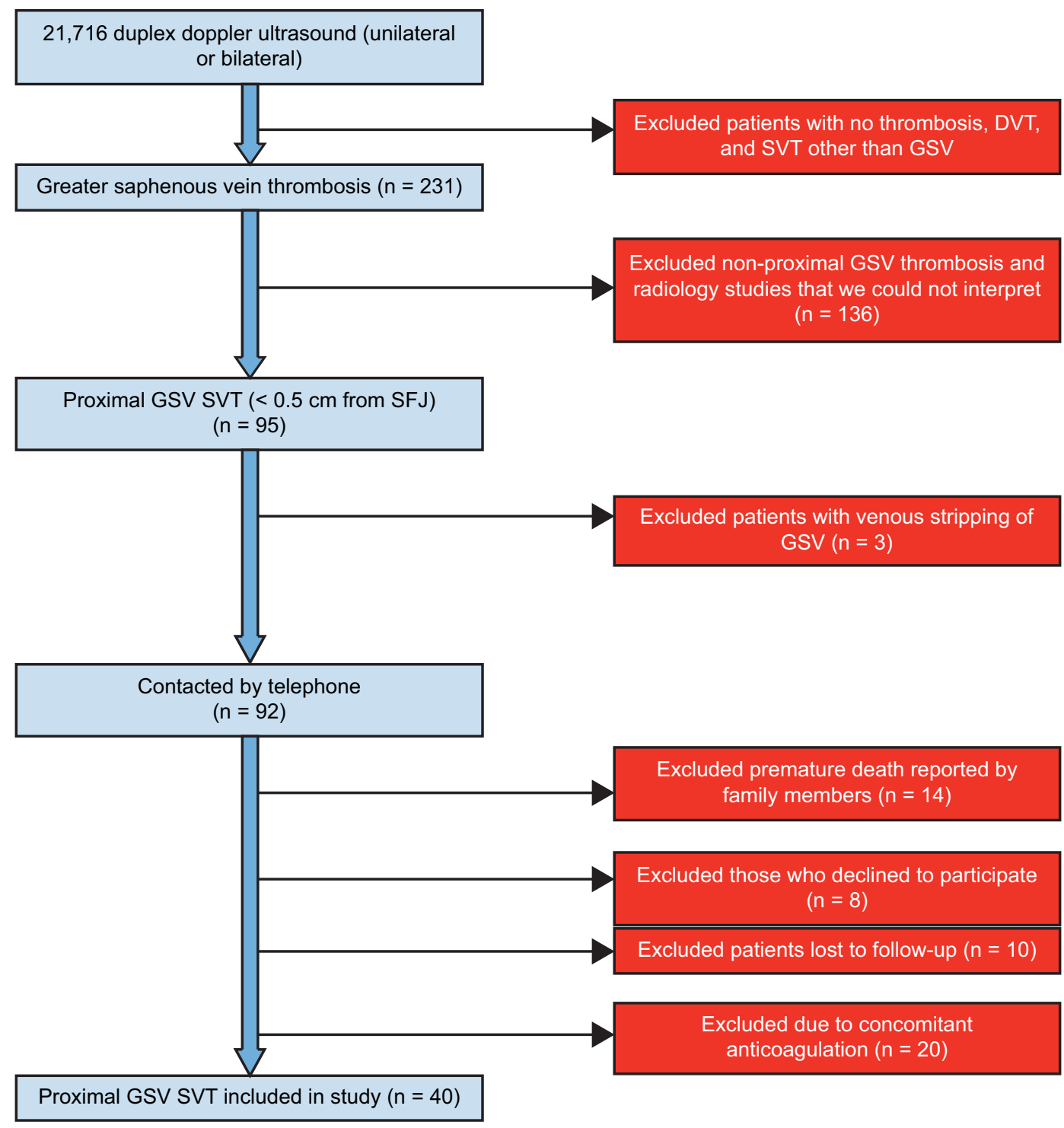

Figure 2 Patient selection process.

Abbreviations: DVT, deep vein thrombosis; GSV, greater saphenous vein; SVT, superficial venous thromobphlebitis; SF], saphenofemoral junction.

removing 21,676 exclusions, the final data set included 40 study subjects.

Patients eligible for this study met these criteria: age $>18$ years and superficial venous thrombosis in the proximal GSV on lower extremity duplex Doppler venous ultrasonography. Patients were excluded based on those not meeting inclusion criteria, those taking anticoagulants at time of ultrasound, those with concomitant DVTs, PEs, and SVTs, those with incomplete lower extremity Doppler at the time of evaluation, and those with history of vein stripping procedures. Additionally, non-English speakers who required a phone script for follow-up, patients with cognitive and hearing impairment, and patients who died $<1$ year after the initial lower extremity Doppler unless diagnosed with DVT/PE were excluded from the study.
Investigators recorded a detailed account of each patient including smoking status, recent surgeries, oral contraceptives, hormone replacement therapy, known malignancy, anticoagulation status, and the use of supportive therapies. Furthermore, subsequent clot extensions, DVTs, and PEs within 1 year of SVT diagnosis in the GSV were collected by reviewing additional ultrasounds, $\mathrm{CT}$, ventilation/perfusion scan data, or from physician notes.

Lower extremity duplex Doppler ultrasonography studies were used to detect thrombosis of the GSV and DVT sequelae. Researchers reviewed radiology interpretations as well as reviewed ultrasound imaging to identify the precise location of the SVT as it relates to the GSV.

To determine patient DVT and PE progression, researchers adhered to a strict phone script protocol while contacting 
patients for follow-up data. Information not found in patient charts and through EPIC, such as past medical history, DVT risk factors, cast applications, and surgeries, were collected from patients directly and/or from their primary care physician.

The Institutional Review Board approves protocols for any investigation involving humans or animals and that all investigations were conducted in conformity with the protocol, and the ethical and humane principles of research. Specifically, written informed consent was deemed unnecessary as this was a retrospective study with minimal risk to patients. A verbal consent and volunteer participation was obtained before data was collected, and patients with concerns or questions were referred to the Institution Review Board.

Descriptive statistics were applied to these subjects, and nominal data was extracted from chart review of EPIC and analysis with SAS for Windows version 9.3, Cary, NC.

\section{Analysis/results}

Forty patients were included in the study. Fifty-five percent of patients were female. Table 1 summarizes patient population characteristics. Three patients $(7.5 \%)$ in our study progressed - two progressing to DVT and one subsequently developing both DVT and PE - within 1 year of SVT diagnosis.

The main secondary outcomes focused on practitioner practice patterns and role of anticoagulation in treatment of proximal GSV SVT. Twenty percent of patients without malignancy were treated with anticoagulation as compared to $14 \%$ of patients with preexisting malignancy. Irrespective of preexisting malignancy, only one of the four of these patients subsequently underwent anticoagulation. Forty-five percent

Table I Patient characteristics $(n=40)$

\begin{tabular}{ll}
\hline $\begin{array}{l}\text { Mean age } \\
\text { Gender }\end{array}$ & 65 (range: 34-93) \\
Male & $18(45 \%)$ \\
Female & $22(55 \%)$ \\
Malignancy & $14(35 \%)$ \\
Subsequent PE & $1(2.5 \%)$ \\
Subsequent DVT & $3(7.5 \%)$ \\
Current tobacco smoker & $20(50 \%)$ \\
Former tobacco smoker & $1(2.5 \%)$ \\
Oral contraceptives & $1(2.5 \%)$ \\
HRT & $1(2.5 \%)$ \\
Surgery within 30 Days & $7(17.5 \%)$ \\
Anticoagulation & $7(17.5 \%)$ \\
Supportive therapy & $18(45 \%)$
\end{tabular}

Notes: anticoagulation included novel oral anticoagulation, Warfarin with subcutaneous heparin or subcutaneous heparin only. 'Supportive therapies included leg elevation, pain medication, hot/cold compresses, monitoring, follow-up with primary care physician, and encouraging ambulation.

Abbreviations: DVT, deep vein thrombosis; HRT, hormone replacement therapy; $\mathrm{PE}$, pulmonary embolism. of patients were treated with supportive therapy for SVT. Treatment patterns for supportive therapy also had considerable variation with common recommendations including leg elevation, pain medication, hot/cold compresses, and provider recommended ambulation. The diagnosis was not specifically treated in more than half of all patients.

\section{Discussion}

SVT is commonly encountered diagnosis affecting 3\%-11\% of the general population. ${ }^{12,13}$ The goal of this paper was to evaluate isolated SVT specifically located at the proximal GSV, which is a much rarer finding with only $0.4 \%(95 / 21716)$ of our cohort receiving this diagnosis. ${ }^{24}$ Comparison of our study group with the existing literature is challenging as preceding studies have not used similar patient inclusion/ exclusion criteria. Some studies have included patients with concurrent DVT and SVT, while others have focused on all locations of SVT. As the primary goal of this study was to focus on the role of the proximal GSV SVT in isolation in progression to DVT or PE, we eliminated all patients with concomitant DVT and distal SVTs.

Three patients $(7.5 \%)$ in our study group experienced propagation of clot to DVT or PE (one patient developed both PE and DVT). A large 2014 Danish study by Canneigieter et al suggests rates are similar to our findings at $10.66 \%$ rate of DVT. ${ }^{24}$ This study had broader inclusion criteria and examined SVT in general. Other studies have cited higher rates. . $^{3,13,14,20,25}$ Though not confined to the GSV, one prospective cohort study of 60 patients with symptoms concerning SVT found 17 participants developed PE (28.3\%) and eight had DVT (13.3\%).

Fourteen patients in our cohort had preexisting malignancy. Malignancy was an important risk factor in patients that ultimately had propagation to DVT and PE. Two of the three subjects who developed DVT were diagnosed with malignancy. In the only case of PE progression, the patient was known to have a history of both liver and skin cancer. Interestingly, only $14 \%$ of patients with history of malignancy were treated with anticoagulation after diagnosis of proximal GSV SVT compared to $20 \%$ of patients without history of malignancy. Our research suggests that this may be a subgroup with proximal GSV SVT that may benefit from anticoagulation.

Half of our study subjects had a history of smoking. Among the three patients with progression, two either had a smoking history or were actively smoking. The patient with both PE and DVT progression had a particularly heavy smoking history (60 pack-year history). The role of cigarette smoking in the development of venous thromboembolism 
(VTE) is somewhat unclear, although an association is possible. Data both for and against smoking as an independent risk factor in VTE exist in the literature. ${ }^{26,27}$ A recent metaanalysis of 9 prospective studies concludes that smoking is not associated with increased VTE risk, however, when further analyzing subtypes, it may yet still play a role in provoked VTE. ${ }^{27}$ Further research is needed on this topic to better define the true risk.

Recent surgery, which has also been closely examined for risk of PE and is a well-established risk factor for development of DVT, was examined in our study. ${ }^{28,29}$ Seven (17.5\%) of those patients identified with isolated proximal GSV had had recent surgery (within the last 30 days) in our study; however, none of those with progression were included among those patients.

The high rate of propagation of proximal GSV SVTs is concerning, and we aimed to better understand practice patterns for this disease process and describe the role of anticoagulation. While supportive measures were frequently employed among patients, specific interventions and combinations tended to vary widely with no commonly accepted regimen among our study population. Supportive measures for the treatment of SVT are common practice and may include single or combination therapy including but not limited to aspirin, warm/cold compresses, sequential compression devices, and encouraging ambulation. ${ }^{18}$ Due to the retrospective design, it was not always clear from the medical record if the patient was receiving these therapies and to what degree they were employed. Other studies have evaluated the role of supportive therapies as a treatment modality. Similar to our study, it is unclear that any specific intervention improves outcomes. Further, we found the variation in practice with supportive therapies, which further underscores the lack of consensus among practitioners regarding the treatment of SVT.

The role of anticoagulation in the treatment of proximal GSV SVT was explored in this study. Nearly $18 \%$ of our study subjects were treated with anticoagulants. Among the three patients with progression to DVT/PE, only one patient was started on anticoagulation at the time of isolated proximal GSV SVT diagnosis.

\section{Limitations}

Limited sample size complicated our study, a difficulty frequently encountered by many of the previous studies into SVTs in general. Indeed, while reviewing available prospective studies, there was consistently a difficulty when attempting to establish clear statistical significance, especially due to the relative rarity of isolated proximal GSV SVT. ${ }^{25,30,31}$
Another limitation of this study was the inability to track follow-up data on eighteen patients who either could not be reached by telephone follow-up or refused to participate. These patients were diagnosed with isolated proximal GSV SVT but had limited information available in our medical record and propagation of thrombosis could not be assessed. For the ten patients that could not be reached, study investigators attempted multiple phone calls and left voicemails per study protocol but were ultimately unsuccessful.

\section{Conclusion}

The risk of propagation of isolated proximal GSV SVT is significant, and practitioners should consider it when making treatment decisions. Anticoagulation is a commonly used treatment option for this condition although the risks and benefits of this therapy were not specifically assessed in this study. Supportive measures are a commonly employed therapy with no clear consensus that one measure has superior outcomes to another. As isolated proximal GSV SVT has a relatively low incidence, further prospective multicenter trials are needed to explore this topic to determine true statistical significance and establish our suspected relevance to widespread clinical practice.

\section{Author contributions}

We had full access to all the data in the study, take responsibility for the integrity of the data and the accuracy of the data analysis, as well as the decision to submit for publication. All authors contributed toward data analysis, drafting and revising the paper and agree to be accountable for all aspects of the work.

\section{Disclosure}

The authors report no conflicts of interest in this work.

\section{References}

1. Bergqvist D, Jaroszewski H. Deep vein thrombosis in patients with superficial thrombophlebitis of the leg. BMJ. 1986;292:658-659.

2. Fesmire F, Brown M, Espinosa J, et al. Critical issues in the evaluation and management of adult patients presenting to the emergency department with suspected pulmonary embolism. Ann Emerg Med. 2011;57:628-652.e75.

3. Goldhaber S, Bounameaux H. Pulmonary embolism and deep vein thrombosis. Lancet [Internet]. 2012 [cited December 21, 2017];379:1835-1846. Available from: http://www.sciencedirect. com/science/article/pii/S0140673611619041?via\%3Dihub. Accessed January 15, 2018.

4. Kearon C, Akl EA, Comerota AJ, et al. Antithrombotic therapy for VTE disease: Antithrombotic Therapy and Prevention of Thrombosis, 9th ed: American College of Chest Physicians Evidence-Based Clinical Practice Guidelines. Chest. 2012;141(2 Suppl):e419S-e496S. 
5. Sobreira ML, Maffei FH, Yoshida WB, et al. Prevalence of deep vein thrombosis and pulmonary embolism in superficial thrombophlebitis of the lower limbs: prospective study of 60 cases. Int Angiol. 2017;28:400-408.

6. Thaler J, Pabinger I, Ay C. Anticoagulant treatment of deep vein thrombosis and pulmonary embolism: the present state of the art. Front Cardiovasc Med. 2015;2:30.

7. De Weese M. Current Therapy in Vascular Surgery. 2nd ed. Philadelphia, PA: BC Decker; 1992.

8. Hanson J, Ascher E, DePippo P, et al. Saphenous vein thrombophlebitis (SVT): A deceptively benign disease. J Vasc Surg. 1998;27:677-680.

9. Subramaniam P, Van Doornum S. Superficial thrombophlebitis: underlying hypercoagulable states. ANZ J Surg. 1999;69:461-463.

10. Litzendorf M, Litzendorf M. Superficial venous thrombosis: disease progression and evolving treatment approaches. Vasc Health Risk Manag. 2011;7:569-575.

11. Pomero F, Di Minno MN, Tamburini Premunian E, et al. A clinical score to rule out the concomitant presence of deep vein thrombosis in patients presenting with superficial vein thrombosis: The ICARO study. Thromb Res. 2015;136:938-942.

12. Decousus H, Leizorovicz A. Superficial thrombophlebitis of the legs: still a lot to learn. J Thromb Haemost. 2005;3:1149-1151.

13. Leon L, Giannoukas A, Dodd D, Chan P, Labropoulos N. Clinical significance of superficial vein thrombosis. Eur J Vasc Endovasc Surg. 2005;29:10-17.

14. Di Minno M, Ambrosino P, Ambrosini F, Tremoli E, Di Minno G, Dentali F. Prevalence of deep vein thrombosis and pulmonary embolism in patients with superficial vein thrombosis: a systematic review and meta-analysis. J Thromb Haemost. 2016;14:964-972.

15. Di Minno G, Mannucci P, Tufano A, et al. The first ambulatory screening on thromboembolism: a multicentre, cross-sectional, observational study on risk factors for venous thromboembolism. J Thromb Haemost. 2005;3:1459-1466.

16. Aper T, Simanowski J. Diagnostic and Surgical Treatment of the Thrombophlebitis in the Saphenofemoral Junction. Ultraschall Med. 2009;30:180-184.

17. Sover E, Brammer H, Rowedder A. Thrombosis of the proximal greater saphenous vein: ultrasonographic diagnosis and clinical significance. J Ultrasound Med. 1997;16:113-116.

18. Marchiori A, Mosena L, Prandoni P. Superficial vein thrombosis: risk factors, diagnosis, and treatment. Semin Thromb Hemost. 2006;32:737-743.

19. Blumenberg R, Barton E, Gelfand M, Skudder P, Brennan J. Occult deep venous thrombosis complicating superficial thrombophlebitis. J Vasc Surg. 1998;27:338-343.

20. Chengelis D, Bendick P, Glover J, Brown O, Ranval T. Progression of superficial venous thrombosis to deep vein thrombosis. J Vasc Surg. 1996;24:745-749.

21. Frappé P, Buchmuller-Cordier A, Bertoletti L, et al. Annual diagnosis rate of superficial vein thrombosis of the lower limbs: the STEPH community-based study. J Thromb Haemost. 2014;12:831-838.

22. Labropoulos N, Bishawi M, Gasparis A, Tassiopoulos A, Gupta S. Great saphenous vein stump thrombosis after harvesting for coronary artery bypass graft surgery. Phlebology. 2012;29:215-219.

23. Verlato F, Zucchetta P, Prandoni P, et al. An unexpectedly high rate of pulmonary embolism in patients with superficial thrombophlebitis of the thigh. J Vasc Surg. 1999;30:1113-1115.

24. Cannegieter SC, Horvath-Puho E, Schmidt M, et al. Risk of venous and arterial thrombotic events in patients diagnosed with superficial vein thrombosis: a nationwide cohort study. Blood. 2014;125:229-235.
25. Hirmerova J, Seidlerova J, Subrt I. Deep vein thrombosis and/or pulmonary embolism concurrent with superficial vein thrombosis of the legs: cross-sectional single center study of prevalence and risk factors. Int Angiol. 2013;32:410-416.

26. Cheng Y, Liu Z, Yao F, et al. Current and former smoking and risk for venous thromboembolism: a systematic review and meta-analysis. PLoS Med. 2013;10:e1001515.

27. Mahmoodi B, Cushman M, Anne Næss I, et al. Association of traditional cardiovascular risk factors with venous thromboembolism clinical perspective. Circulation. 2016;135:7-16.

28. Kline J, Mitchell A, Kabrhel C, Richman P, Courtney D. Clinical criteria to prevent unnecessary diagnostic testing in emergency department patients with suspected pulmonary embolism. J Thromb Haemost. 2004;2:1247-1255.

29. Wells P, Anderson D, Rodger M, et al. Excluding pulmonary embolism at the bedside without diagnostic imaging: management of patients with suspected pulmonary embolism presenting to the emergency department by using a simple clinical model and d-dimer. Ann Intern Med. 2001;135:98-107.

30. Decousus H, Quere I, Presles E. Superficial venous thrombosis and venous thromboembolism: a large, prospective epidemiologic study. J Vasc Surg. 2010;52:1727-1728.

31. Prandoni P, Tormene D, Pesavento R. High vs. low doses of lowmolecular-weight heparin for the treatment of superficial vein thrombosis of the legs: a double-blind, randomized trial. J Thromb Haemost. 2005;3:1152-1157.

32. Decousus H, Prandoni P, Mismetti P. Fondaparinux for the treatment of superficial-vein thrombosis in the legs. JVasc Surg. 2011;53:549-550.

33. Enoxaparin Study Group. A pilot randomized double-blind comparison of a low-molecular-weight heparin, a nonsteroidal anti-inflammatory agent, and placebo in the treatment of superficial vein thrombosis. Arch Intern Med. 2003;163:1657-1663.

34. Krause U, Kock H, Kroger K, Albrecht K, Rudofsky G. Prevention of deep vein thrombosis associated with superficial thrombophlebitis of the leg by early saphenous vein ligation. Vasa. 1998;27:34-38.

35. Lee J, Cooke J. The role of nicotine in the pathogenesis of atherosclerosis. Atherosclerosis. 2011;215:281-283.

36. Massachusetts General Hospital. DVT: What you should know [Internet]. Massachusetts General Hospital; 2017 [cited December 5, 2017]. Available from: http://www.massgeneral.org/News/newsarticle. aspx?id=1603. Accessed January 15, 2018.

37. Prountjos P, Bastounis E, Hadjinikolaou L, Felekuras E, Balas P. Superficial venous thrombosis of the lower extremities co-existing with deep venous thrombosis: a phlebographic study on 57 cases. Intern Angiol. 1991;10:63-65.

38. Quenet S, Laroche J, Bertoletti L, et al. Value of a planned compression ultrasonography after an isolated superficial vein thrombosis: results from a prospective multicentre study. Eur J Vasc Endovasc Surg. 2012;43:233-237.

39. Schönauer V, Kyrle P, Weltermann A, et al. Superficial thrombophlebitis and risk for recurrent venous thromboembolism. $J$ Vasc Surg. 2003;37:834-838.

40. Skillman J, Kent K, Porter D, Kim D. Simultaneous occurrence of superficial and deep thrombophlebitis in the lower extremity. $J$ Vasc Surg. 1990;11:818-824.

41. Van Langevelde K, Lijfering W, Rosendaal F, Cannegieter S. Increased risk of venous thrombosis in persons with clinically diagnosed superficial vein thrombosis: results from the MEGA study. Blood. 2011;118: 4239-4241. 


\section{Publish your work in this journal}

Vascular Health and Risk Management is an international, peerreviewed journal of therapeutics and risk management, focusing on concise rapid reporting of clinical studies on the processes involved in the maintenance of vascular health; the monitoring, prevention and treatment of vascular disease and its sequelae; and the involvement of metabolic disorders, particularly diabetes. This journal is indexed on PubMed Central and MedLine. The manuscript management system is completely online and includes a very quick and fair peer-review system, which is all easy to use. Visit http://www.dovepress.com/ testimonials.php to read real quotes from published authors.

Submit your manuscript here: https://www.dovepress.com/vascular-health-and-risk-management-journal 